\title{
CONCEPTION OF A SCALABLE PRODUCTION FOR MICRO-MECHATRONICAL PRODUCTS
}

Systematics for planning and platform with process modules for the production of micro-mechatronical products

Jürgen Fleischer, Luben Krahtov, Torsten Volkmann

wbk - Institute of Production Science, University of Karlsruhe, Germany

\begin{abstract}
Miniaturization and mechatronics are important technological trends of the last years and will be gaining importance in the future. The micro-mechatronical products which have already been put on the market are currently designed to meet the abilities of existing production techniques and are manufactured as mass products in specialized and capital intensive plants. In order to make miniaturized mechatronical systems more attractive for the market, their production must be made possible in small and medium volumes at acceptable costs. A holistic approach for the conception of a scalable micro production which consists of the development of an integrated planning process as well as the creation of a flexible production platform with general process modules is presented and demonstrated considering as example the realization of a scalable production for a micro-mechatronical actuator. The approach is characterized by a high level of flexibility considering joining technologies as well as small space requirements. A reduction of capital costs for production technology and a stepwise extension of the production are achieved.
\end{abstract}

Key words: Planning Systematics, Micro-Assembly, Micro-Mechatronical Products, Automation

\section{INTRODUCTION}

In the last years, an important market with considerable growth rates and a large number of different products has developed in the field of micro technology ${ }^{1,2,3}$. The main application areas are the information and communications technology, automation, automotive and medical engineering ${ }^{4}$. The production methods essentially being used for manufacturing of micro-products can be subdivided into two differing 
procedures. On the one hand, there is micro-systems engineering based on lithographical processes, which developed out of the domain of semiconductor engineering. On the other hand, there is precision engineering where classical manufacturing methods are used to produce parts with increasingly smaller dimensions.

For manufacturing micro-mechatronical products, both the manufacturing methods of the micro-systems technology and the precision engineering are applied ${ }^{5}$. In this context micro-assembly plays an important role in integrating the variously produced components into a complete system. Micro-assembly is characterized by high requirements in accuracy and constraints in package dimension. Numerous and differing joining processes, which are very complex due to the micro-specific requirements, e.g. small influence of gravitational forces in comparison with surface forces, high sensitivity and small tolerances of the components, are applied. The micro-mechatronical products discussed in this paper are composed three dimensionally and the component size is within the range of $400 \mu \mathrm{m}$ up to $4 \mathrm{~mm}$ and the total product size within the range of $2 \mathrm{~mm}$ up to $50 \mathrm{~mm}$.

The selection and verification of the micro-assembly processes are very extensive since there is a deficit of a holistic planning systematics. As a result, a long product introduction phase which takes a significant portion of the total product life cycle is caused. At present, most micro-technical products are manufactured as mass products in specialized and capital intensive plants ${ }^{6}$. Production of miniaturized mechatronical systems in small to medium volumes, which is mostly performed manually, is restricted due to complexity and quality requirements. In order to make miniaturized mechatronical systems increasingly more attractive for the market, production must be enabled in small and medium volumes at acceptable prices ${ }^{7}$. Currently, there are only a few solutions which permit an economical access to micro production and suit for a scalable extension or a reconfiguration.

The Institute of Production Science at the University of Karlsruhe is elaborating a holistic approach for the conception of a scalable micro production which consists of the development of an integrated planning process as well as the creation of a flexible production platform with general process modules ${ }^{8,9,10}$.

\section{INTEGRATED PLANNING PROCESS}

The input of the integrated planning systematics (see Fig. 1) is the structure of a micro-mechatronical product. The selection of processes for micro-assembly is supported by a feature-based procedure. A micro- 
assembly precedence graph is derived and contains all technically possible solution alternatives of the assembly for the product. Due to micro-specific requirements, e.g. gripper selection considering adhesion behavior or sensitivity of the components, the micro-assembly precedence graph includes micro-specific information fields. This ensures that assembly sequence as well as information of handling steps and linkage of the single processes is contained. Based on this micro precedence graph, linkage concepts, e.g. small volume manufacturing with a stand alone system, series manufacturing in linked systems, are developed. By means of the simulation tool Tecnomatix eM-Plant, which is adapted, the concepts will be evaluated and improved in an iteration loop. As a result of the planning systematics, an optimized and configured assembly is provided.

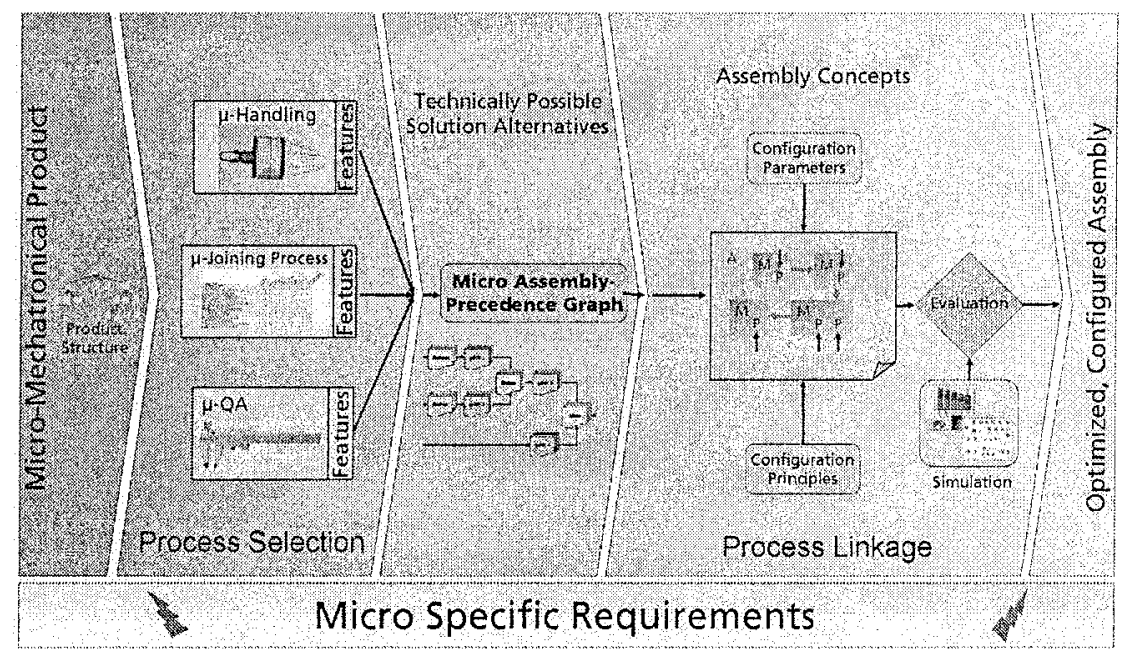

Figure l. Integrated planning systematics for micro-mechatronical products

\subsection{Selection of Processes and Handling Tools}

The selection of processes and handling tools plays an important role within the planning of micro-assembly. Micro-assembly can be divided into two basic operations: joining processes as well as the appropriate handling steps. Between each joining process, a handling operation comprising mainly gripping procedures, but also the transport of components from one process step to the next, is applied. These handling operations should be integrated into the planning process like all other process steps. To plan the micro handling processes a method based on feature technology is developed. 
Complex handling operations like flipping or joining of miniaturized components must be planned in detail due to the interference effects of the micro world, e.g. adhesive forces. As a consequence of the insufficiently examined effects of micro handling, the planning of these processes is difficult. It is quite impossible to make exact predictions about the handling of micro components until it is tested in empirical studies. Thus, in the earlier phase, when physical micro components are not available, exact planning of micro-assembly processes is impossible.

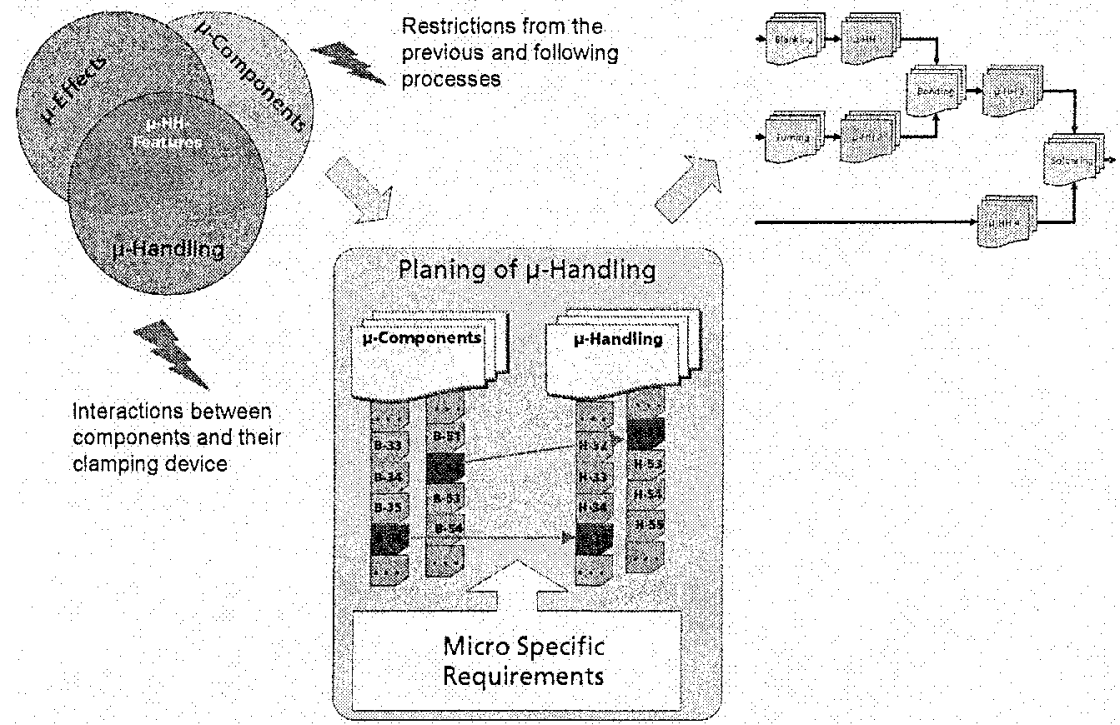

Figure 2. Derivation of the micro-assembly precedence graph with feature technology

An important precondition for the holistic planning process is the removal of these planning deficits by creating a methodology for the description of micro components, micro interference effects and the micro handling tools by feature-technology. The method (see Fig. 2) conjugates features (e.g. sensitivity) to the components and performs a comparison with the corresponding gripper features to determine the compatibility. In addition to geometrical information the micro handling features contain semantic information (e.g. tendency of adhesion), restrictions from the previous and following processes as well as interactions between components and their clamping device. The micro handling features are the logical succession of the construction, manufacturing and quality assurance features ${ }^{11,12}$. By applying feature technology in the planning phase of the product, the accurate assignment of components to a suitable gripper with 
regard to the corresponding environmental restrictions becomes possible. As described above a detailed product specific micro-assembly precedence graph is derived in which all assembly processes are planned in detail.

\subsection{Process Linkage}

Based on the micro-assembly precedence graph, a linkage concept is elaborated. A methodology to provide concepts depending on a rule-based selection is developed. Rules considering technical, economic and logistical design parameters and principals as well as input and output relations and interactions have to be defined. An important requirement on the concepts is an accelerated cost and risk minimized realization of the production with regard to varying production volumes. The stepwise extension is supported by the integrated planning systematics and in each case the optimal configuration is determined. As a basis for the planning systematics, a flexible production platform and standardized process and linkage modules are required to set up a scalable production for micro-mechatronical systems. In research there exist only a few approaches which are related to the manufacturing of sensors and biological chips ${ }^{13}$. However, no integrated planning in terms of a scalable production is taken into consideration.

An important criterion for a scalable production is to use the production equipment of the development and prototype phase also for the production of small to medium volumes. Particularly at a production within this range an unsorted supply of single components with picking and orienting is difficult and expensive to realize or even not applicable. This is caused by the sensitivity against mechanical damage and adhesive problematic of the micro components. Especially in bonding processes the components must be cleaned and pretreated. For this reason, the concept of a work piece carrier is used as a solution approach. A work piece carrier which passes through the entire production can be designed according to different technical and logistical constraints. Numerous functions can be performed by the work piece carrier including storage of raw materials, hybrid 3-dimensional microcomponents and tools as well as additional and auxiliary axes or gripping and clamping devices. The work piece carrier integrates tasks of the assembly area, the devices and the transportation medium in itself. A direct assembly on the work piece carrier is easy to realize and leads to shorter assembly time and better achievable accuracies because this process requires less space. The tolerances of the work piece carrier are compensated by an image processing system of the assembly platform and a high joining accuracy is ensured. The transport of the components between different processes or machines is reliable with the work piece carrier and is 
simplified by providing the once achieved orientation of the components and the standardized interface. In addition, components and assembly relevant data can be easily assigned and traced by a identification tag at the work piece carrier.

\section{MODULAR PLATFORM AND PROCESS MODULES}

The production of micro-mechatronical products in small to medium volumes plays an important role for the investment in the production equipment. Automated solutions are mostly dimensioned for high quantities and comprise special solutions for one product with several machines and expensive linkage technology. An approach for the economical access to automated micro-assembly is to start automation with a modular platform, process modules and work piece carrier linkage.

The platform makes an economical basic infrastructure available for the micro-assembly. An axis system which can reach all positions within the work space and offers a tool or gripper fitting is provided for. For precise positioning of the axes a high definition image processing system is applied and allows high flexibility. Furthermore, an open control concept makes the integration of the process modules quick and easy to integrate (CAN-Open).

The required processes in micro-assembly are integrated in general process modules, e.g. positioning, rotating, pressing, riveting, adhesive / soldering paste dispensing, heat hardening / UV exposing, ultrasonic / laser welding or quality assurance.

The platform concept enables an easy upgrading and multiplication of the production and a stepwise extension can be fulfilled with relatively equal processes. While within the pilot phase the focus is on the verification of the processes and the determination of the necessary process parameters, the commissioning and the flow of material become increasingly important as the production quantity increases. With this concept, the components can initially be cleaned manually, sorted, and then commissioned on the work piece carrier for a small volume production. In further stages of development, the commissioning can be performed automatically by commissioning devices. Furthermore, the process modules can be distributed on multiple machines and linked by appropriate linkage modules to increase the productivity.

The advantage of this proceeding is that the assembly processes are used from the first phase up to the expansion to a production of large-scale volumes in an automated environment. Thus, it is possible to identify the manufacturing, assembly, material allocation and the logistical problems at 
an early stage. Beyond that, the modular platform as well as the process modules can be modeled and evaluated with simulation tools.

\section{PRODUCTION OF MICRO-MECHATRONICAL SYSTEMS}

\subsection{Demonstrator Product}

An example of a micro-mechatronical product is a flat motor of the company "mymotors" (diameter $12 \mathrm{~mm}$, thickness $3 \mathrm{~mm}$ ). It is characterized by a complex assembly and has a characteristical mechatronical structure, e.g. mechanical components generate signals and printed circuit boards have mechanical functions.

The product consists of eleven single components and several joining processes are applied for the assembly. As described in chapter 2, the features, e.g. sensitivity of the components were analyzed at first and according to the product structure a micro-assembly precedence graph (see Fig. 3) was derived. It contains all processes, e.g. bonding, soldering, pressing, riveting and describes the linkage between processes, handling and linkage. Furthermore, the micro-specific restrictions are taken into account by designing the handling tools and the work piece carrier.

The layout of the process and linkage modules within the modular production platform is derived out of the micro-assembly precedence graph. As modular platform a micro-assembly machine from the company "Häcker Automation" is applied and described below.

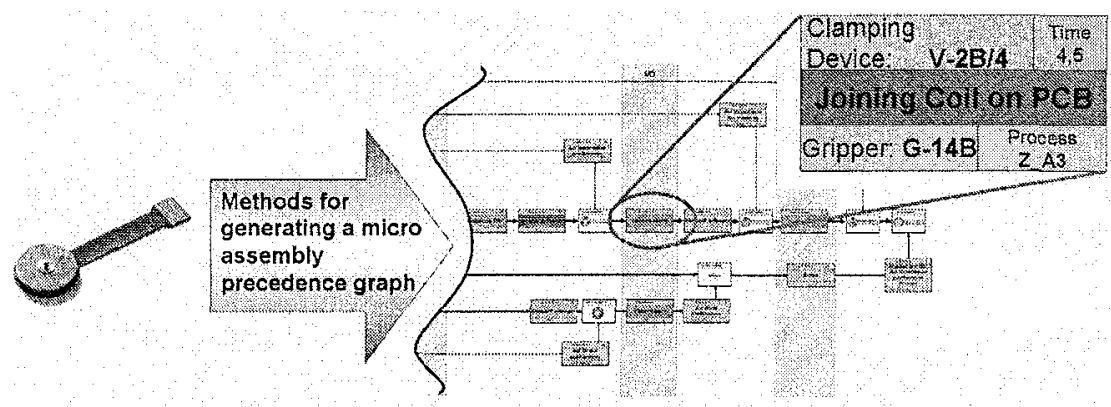

Figure 3. Micro-mechatronical flat motor and corresponding micro-assembly precedence graph 


\subsection{Modular Production Platform on the Example of a Micro-Assembly Machine}

The micro-assembly machine consists of a precise serial kinematics with a repeat accuracy of $20 \mu \mathrm{m}$ and a flexible and modular configurable working space of $450 \times 450 \mathrm{~mm}^{2}$. The placement head integrates an image processing system (IPS) and a double head system.

The IPS is able to percept and analyze objects three dimensionally. Two high-resolution cameras are used and positioned at an angle of approximately $23^{\circ}$ in relation to one another. Thus, the system is able to locate the position of the components as well as to detect their height with an accuracy down to $5 \mu \mathrm{m}$.

The double head system consists of a placement head and a dispense head. The placement head has a vacuum connection and an integrated gripper change module which enables a fully automated change of various vacuum and mechanical grippers. An innovative measure system (spatiovolumetric flow rate) enables the dispense head to perform dosings of media with different viscosities with an accuracy in the dimension of picoliters. After each dispense procedure a correction of the time-pressure control is done in correlation to the quantity dispensed during the previous procedure. Changes in viscosity of the different media are actively adjusted.

In addition to these permanently installed components, several other automated process modules, which can be placed in the working area, are available (see Fig. 4). These are, e.g. a flipping station or a 3D-station with additional axes for flipping. Furthermore, a heat lamp was developed as a process module to integrate bonding and soldering processes.

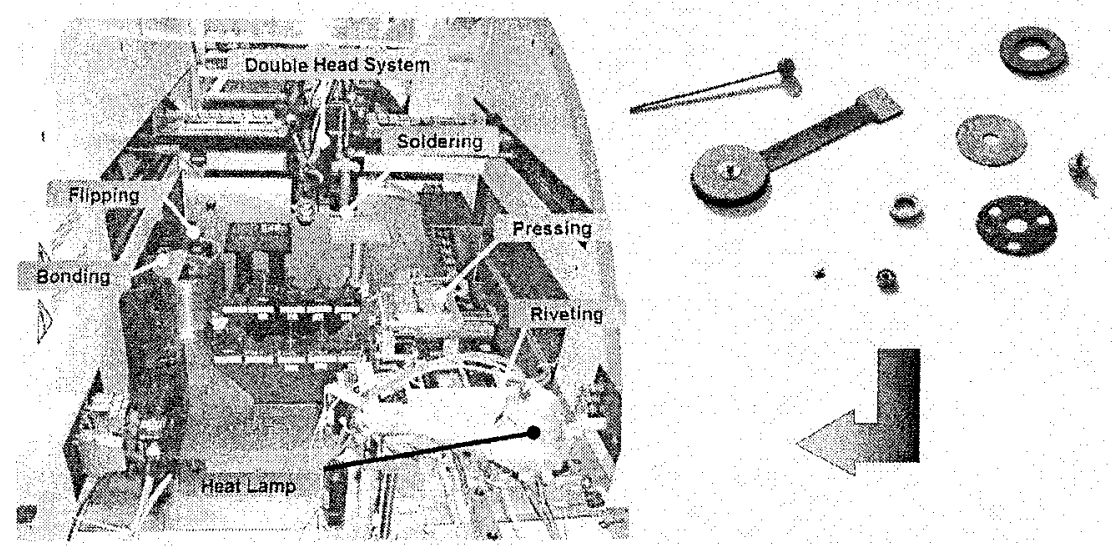

Figure 4. Micro-assembly machine with double head system 


\subsection{Process Modules on the Example of a combined Soldering, Bonding and Pressing Module}

The two joining processes of bonding and soldering are playing a substantial role in the assembly of the flat motor. The rapid curing of adhesive or melting of soldering paste at a defined time within the assembly process is important for the automation of the process. A small and compact heat source which is characterized by a fast selective and local heating area is necessary. As a solution, a heat lamp was developed. It consists of a reflector, a halogen lamp and a case with heat sink and protection glass. The reflector is a bisected ellipsoid mirror and the halogen lamp is positioned in the first focal point. In the second focal point of the ellipsoid mirror the spot of the bundled light is located and heat is generated. The cooling strategy plays an important role when designing of the heat lamp. The interior of the ellipsoid and the heat sink is streamed and cooled by air. Subsequently, the air is conveyed in front of the protection glass towards the light spot to blow away steam from soldering. Visualization and control of the soldering and bonding processes are important for automation. Two laser diodes permit the accurate positioning of the focal point when teaching in the process. Thus, the $\mathrm{x}-, \mathrm{y}$ - and $\mathrm{z}$-position for the components to be soldered or bonded could be easily determined. Control of the temperature is measured by a laterally attached contactless infrared thermometer.

The z-axis of the heat lamp module is also used for process integration of riveting within this module, due to heat generation and temperature measurement is contactless. The process module can achieve a maximum press force of $1000 \mathrm{~N}$. The pressing force controlled by a force transducer is placed directly behind the replaceable press punch. Thus, three processes with two basic functionalities are implemented within only one module (see Fig. 5). 


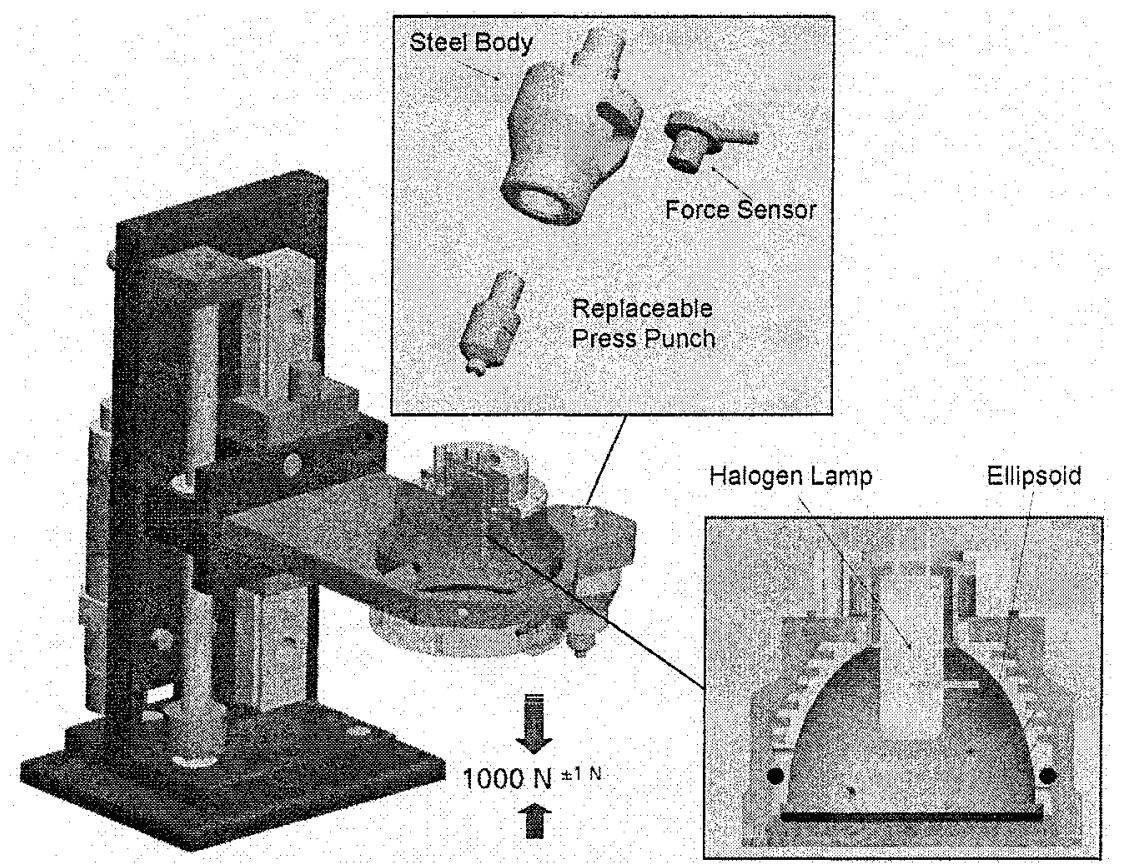

Figure 5. Standardized device with combined soldering, bonding and riveting module

The device is mounted outside the working space of the assembly machine and is supplied by two additional axes. In this way, processes can be executed simultaneously. While performing the soldering process under the heat lamp, the main kinematics of the assembly machine can proceed other operations.

The flexible and modular design of this device offers the implementation of further processes, like e.g. UV-exposure, ultrasonic or laser welding, electronic examination and quality assurance.

\section{LINKAGE WITH WORK PIECE CARRIER ON THE EXAMPLE OF A SMALL VOLUME PRODUCTION}

A small volume production of a flat motor on the micro-assembly machine by using a work piece carrier was implemented at the Institute of Production Science (see Fig. 6). The layout consists of the process modules for bonding and flipping processes, the tool station for replaceable grippers and a bottom side camera, as well as an $x-y$-unit for the transport and 
positioning of the work piece carrier. A multi-functional work piece carrier which is designed according to the German DIN $32561^{14}$ is utilized. At the contacted dispensing of adhesive or soldering paste there is the danger of moving the components out of position or pulling them out of their molding cavity due to strong adhesive forces. A vacuum underneath the components eliminates this problem.

Furthermore, the work piece carrier has the functions of a magazine for the components as well as of the assembly area for all the assembly operations during the manufacturing process. Due to a skillful design of the molding cavities, it is guaranteed that the components remain fixed after each joining and bonding operation. In the phase of a small volume production the components are placed manually on the work piece carrier. With increasing production quantities the work piece carrier can be fitted with components by the assembly system, e.g. by belt feeders. The multifunctional work piece carrier is the central element of the previously described automated micro-assembly and can be adapted to product modifications or changes without having influence on the total assembly system. This guarantees the flexibility and modularity of such a scalable concept.

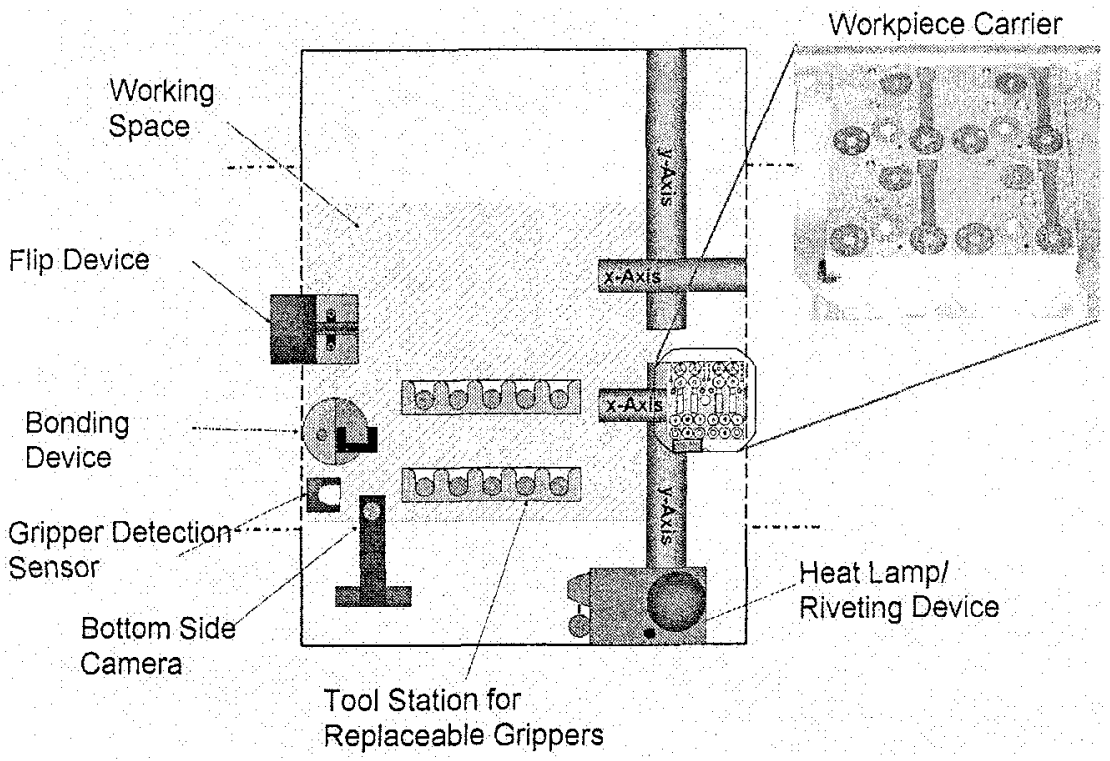

Figure 6. Layout of the automated assembly of the flat motor with multi-functional work piece carrier according to DIN 32561 


\section{SUMMARY}

For the economic manufacturing of micro-mechatronical products, a holistic planning systematics as well as a scalable manufacturing technology plays an increasingly important role. An optimized and configured microassembly is enabled by methodical assistance at the process and handling tool selection phase, the deriving of a micro-assembly precedence graph as well as the selection of a linkage concept. As a basis, a modular assembly platform and a construction kit of process and linkage modules is developed. Thus, a high level of flexibility according to joining technologies, variability and reconfigurability of the system is guaranteed. The manufacturing of initially small volumes and the stepwise extension of production quantities is realized with small space requirements. Due to modular design, the reduction of capital costs for the manufacturing technology is possible and the demand for an economic access into micro manufacturing technology can be achieved.

\section{REFERENCES}

1. W. Ehrfeld, Mikrotechnik - Produktion und Produkte, in: Feinbearbeitung im neuen Jahrtausend - Innovationen und Trends, Internationales Braunschweiger Feinbearbeitungskolloquium (10. FBK), Braunschweig, 2000.

2. J. Hesselbach, mikroPRO - Untersuchung zum internationalen Stand der Mikroproduktionstechnik, Institut für Werkzeugmaschinen und Fertigungstechnik TU Braunschweig, Braunschweig, Vulkan Verlag, 2002.

3. M. Weck; S. Fischer, Maschinenentwicklung für die Mikrotechnik, in: wt Werkstattstechnik 89 (1999), Issue 11/12, p. 489 - 491.

4. H. Van Brussel et al, Assembly of Microsystems, in: Annals of the CIRP Vol. 49/2/2000, p. $451-471$.

5. G. Reinhart, T. Angerer, Automated Assembly of Mechatronic Products, in: Annals of the CIRP Vol. 51/1/2002, p. 1 - 4.

6. D. Zühlke, R.Fischer, J. Hankes, Flexible Montage von Miniaturbauteilen, Düsseldorf, VDI-Verlag, 1997.

7. VDI/VDE-IT, Mikrosystemtechnik in Europa, Teltow, 2001.

8. J. Fleischer, H. Weule, T. Volkmann, Factory Planning Methodology for the Production of Micro-Mechatronical Systems, in Proceedings of the 1st CIRP International Seminar on Micro and Nano Technology, Copenhagen, 2003.

9. J. Fleischer, H. Weule, A. Blessing, T. Volkmann, Factory Planning for Miniaturized Mechatronical Systems, in: Proceedings MICRO.tec 2nd World Micro Technologies Congress, München, 2003.

10. J. Fleischer, T. Volkmann, L. Krahtov, Wirtschaftliche Automatisierung der Mikromontage in der Kleinserienfertigung, in: wt Wetkstattstechnik 94 (2004), Issue 9, p. $390-394$.

11. J. Elsner, Informationsmanagement für mehrstufige Mikro-Fertigungsprozesse, Heinz W. Holler Druck und Verlags GmbH, Karlsruhe, 2003. 
12. DI Richtlinie 2118, Informationsverarbeitung in der Feature-Technologie, Berlin, Beuth Verlag, 2003.

13. T. Gaugel, M. Bengel et al, Building a mini-assembly system from a technology construction kit; in Assembly Automation Band 24, Issue 1; 2004, p. $43-48$.

14. DIN 32561, Production equipment for Microsystems - Tray - Dimensions and Tolerances, Deutsches Institut für Normung e.V., Berlin, 2003. 